\title{
Impact of dispatcher-assisted cardiopulmonary resuscitation and myResponder mobile app on bystander resuscitation
}

Xiang Yi Wong, ${ }_{B S C}$, Qiao Fan, ${ }^{1}{ }_{P h D}$, Nur Shahidah, ${ }_{B A}$, Carl Ross De Souza, ${ }_{B S C}$, Shalini Arulanandam, ${ }^{3}{ }_{M B B S}$, Yih Yng $\mathrm{Ng},{ }^{4,5}{ }_{M B B S}$, Wei Ming $\mathrm{Ng},{ }^{6}{ }_{M B B S}$, Benjamin Sieu-Hon Leong, ${ }^{7} \mathrm{MBBS}$, Michael Yih Chong Chia, ${ }^{5}{ }_{M B B S}$, Marcus Eng Hock Ong, ${ }^{2,8}{ }_{M B B S}$

\begin{abstract}
Introduction: Bystander cardiopulmonary resuscitation (B-CPR) is associated with improved out-ofhospital cardiac arrest survival. Community-level interventions including dispatcher-assisted CPR (DA-CPR) and myResponder were implemented to increase B-CPR. We sought to assess whether these interventions increased B-CPR.

Methods: The Singapore out-of-hospital cardiac arrest registry captured cases that occurred between 2010 and 2017. Outcomes occurring in 3 time periods (Baseline, DA-CPR, and DA-CPR plus myResponder) were compared. Segmented regression of time-series data was conducted to investigate our intervention impact on the temporal changes in B-CPR.

Results: A total of 13,829 out-of-hospital cardiac arrest cases were included from April 2010 to December 2017. Higher B-CPR rates $(24.8 \%$ versus $50.8 \%$ vs $64.4 \%)$ were observed across the 3 time periods. B-CPR rates showed an increasing but plateauing trend. DA-CPR implementation was significantly associated with an increased B-CPR (level odds ratio [OR] 2.26, 95\% confidence interval [CI] 1.79-2.88; trend OR 1.03, 95\% CI 1.01-1.04), while no positive change was detected with myResponder (level OR 0.95, 95\% CI 0.82-1.11; trend OR 0.99, 95\% CI 0.98-1.00).

Conclusion: B-CPR rates in Singapore have been increasing alongside the implementation of community-level interventions such as DA-CPR and myResponder. DA-CPR was associated with improved odds of receiving B-CPR over time while the impact of myResponder was less clear.
\end{abstract}

Ann Acad Med Singap 2021;50:212-21

Keywords: Bystander CPR, community responders, out-of-hospital cardiac arrest, pre-hospital care

\section{INTRODUCTION}

Out-of-hospital cardiac arrest (OHCA) is a leading cause of mortality worldwide, with a global incidence of 62 cases per 100,000 person-years. ${ }^{1}$ In addition, there are variations in the reported survival-to-hospital discharge rates among different regions in the world. In Singapore, OHCA incidence rate was 27.2 per 100,000 personyears, with an overall survival rate of $5.3 \%$ in $2015 .^{2}$
In contrast, some cities had reported higher survival rates, with King County, Seattle in the US reporting a rate of $16 \%,{ }^{1}$ suggesting that more could be done in Singapore to improve OHCA survival.

Key to the treatment of OHCA is the "chain of survival", which comprises early recognition, early cardiopulmonary resuscitation (CPR), early defibrillation, basic and advanced emergency medical

\footnotetext{
${ }^{1}$ Duke-NUS Medical School, Singapore

${ }^{2}$ Department of Emergency Medicine, Singapore General Hospital, Singapore

${ }^{3}$ Medical Department, Singapore Civil Defence Force, Singapore

${ }^{4}$ Lee Kong Chian School of Medicine, Nanyang Technological University, Singapore

${ }^{5}$ Emergency Department, Tan Tock Seng Hospital, Singapore

${ }^{6}$ Emergency Medicine Department, Ng Teng Fong General Hospital, Singapore

${ }^{7}$ Emergency Medicine Department, National University Hospital, Singapore

${ }^{8}$ Health Services and Systems Research, Duke-NUS Medical School, Singapore

Correspondence: Xiang Yi Wong, Duke-NUS Medical School, 8 College Road, Singapore 169857

Email: wongxiangyi@u.duke.nus.edu
} 


\section{CLINICAL IMPACT}

\section{What is New}

- Singapore's cardiac arrest registry study showed that dispatcher-assisted cardiopulmonary resuscitation (CPR) increased odds of bystander CPR (B-CPR). myResponder mobile app did not achieve the same effect.

- An increasing but plateauing trend in B-CPR rates was also noted.

\section{Clinical Implications}

- Our study suggests that dispatcher-assisted CPR has been effective in improving B-CPR rates over time, and supports its continued implementation.

- A plateauing trend in B-CPR rates underscores the need to investigate the optimisation of existing interventions such as myResponder or implementation of other novel interventions.

services (EMS) treatment, and post-resuscitation care. ${ }^{3}$ Survival decreases by $7-10 \%$ per minute without treatment. ${ }^{4}$ CPR carried out by bystanders (B-CPR) has been shown to at least double the chance of survival from $\mathrm{OHCA}^{5}$ and has been recommended to be a global priority. ${ }^{6}$

In Singapore, several new initiatives were introduced in recent years to improve B-CPR rates. In July 2012, dispatcher-assisted CPR (DA-CPR) was implemented at the Singapore Civil Defence Force (SCDF) Operations centre to enable a trained dispatcher to recognise a cardiac arrest over the phone and provide timely instructions to the caller to commence compressiononly CPR. In April 2015, the SCDF launched the myResponder mobile application (app) to recruit and dispatch registered volunteers to potential OHCA cases if they are within a 400-meter radius of the victim. The app also highlights the locations of the nearest public access automated external defibrillators (AEDs) for retrieval by the responders.

The main aim of this study was to assess whether our community-level interventions, including DA-CPR and myResponder, increased the odds of receiving B-CPR compared to baseline prior to their implementation. Secondary aims of the study were to assess the interventions' effect on improving survival outcomes after OHCA. It was hypothesised that our interventions will increase $\mathrm{B}-\mathrm{CPR}$ rates and improve patient survival outcomes.

\section{METHODS}

\section{Setting}

Singapore is a high-density city-state $724.2 \mathrm{~km}^{2}$ in size, with a total population of 5.7 million and a population density of 7,866 per $\mathrm{km}^{2} .7$ Emergency calls are received by the SCDF, which operates a centralised "995" dispatch centre for the country's EMS. A total of 65 ambulances were available for dispatch in $2017 .{ }^{8}$

\section{DA-CPR}

The DA-CPR intervention relies on a standardised dispatch protocol to guide trained dispatch personnel to rapidly and accurately recognise suspected cardiac arrest patients through a systematic "no-no-go" process by posing 2 key questions to callers: Is the patient conscious? Is the patient breathing normally? ${ }^{9}$ Once identified, the protocol also guides the dispatcher on how to instruct the caller to commence CPR. Dispatch instruction is limited to chest compression only, which is associated with better long-term survival compared to traditional CPR that includes ventilation. ${ }^{10}$ Dispatch audio recordings are subsequently reviewed for quality improvement purposes. ${ }^{11}$

\section{myResponder mobile phone application}

myResponder uses the global positioning system (GPS) within mobile phones to locate registered volunteers geographically. Volunteers will need to download the app (available on both Android and iOS platforms), register their names in the system and consent to sharing their GPS location before they can be activated.

When the 995 dispatch centre receives a call for a suspected OHCA, an ambulance (comprising 1 paramedic, 1 emergency medical technician (EMT) and 1 driver) is dispatched to the scene. A fire-biker may also be dispatched when conditions (e.g. availability, weather and traffic) allow. In addition, a 4-man fire vehicle manned by trained EMTs has been dispatched alongside the ambulance since April 2019, as part of a pilot project to introduce a high-performance CPR team. Since the launch of myResponder in April 2015, alerts are also sent out by the dispatch centre to volunteers with an active myResponder app within a 400-metre radius of an OHCA, with the address of the case and the locations of the nearest AEDs. The workflow for the mobile application is illustrated in Fig. 1. Both the number of registered users and the average number of notifications sent for each OHCA case have been steadily increasing since myResponder's implementation in April 2015. 


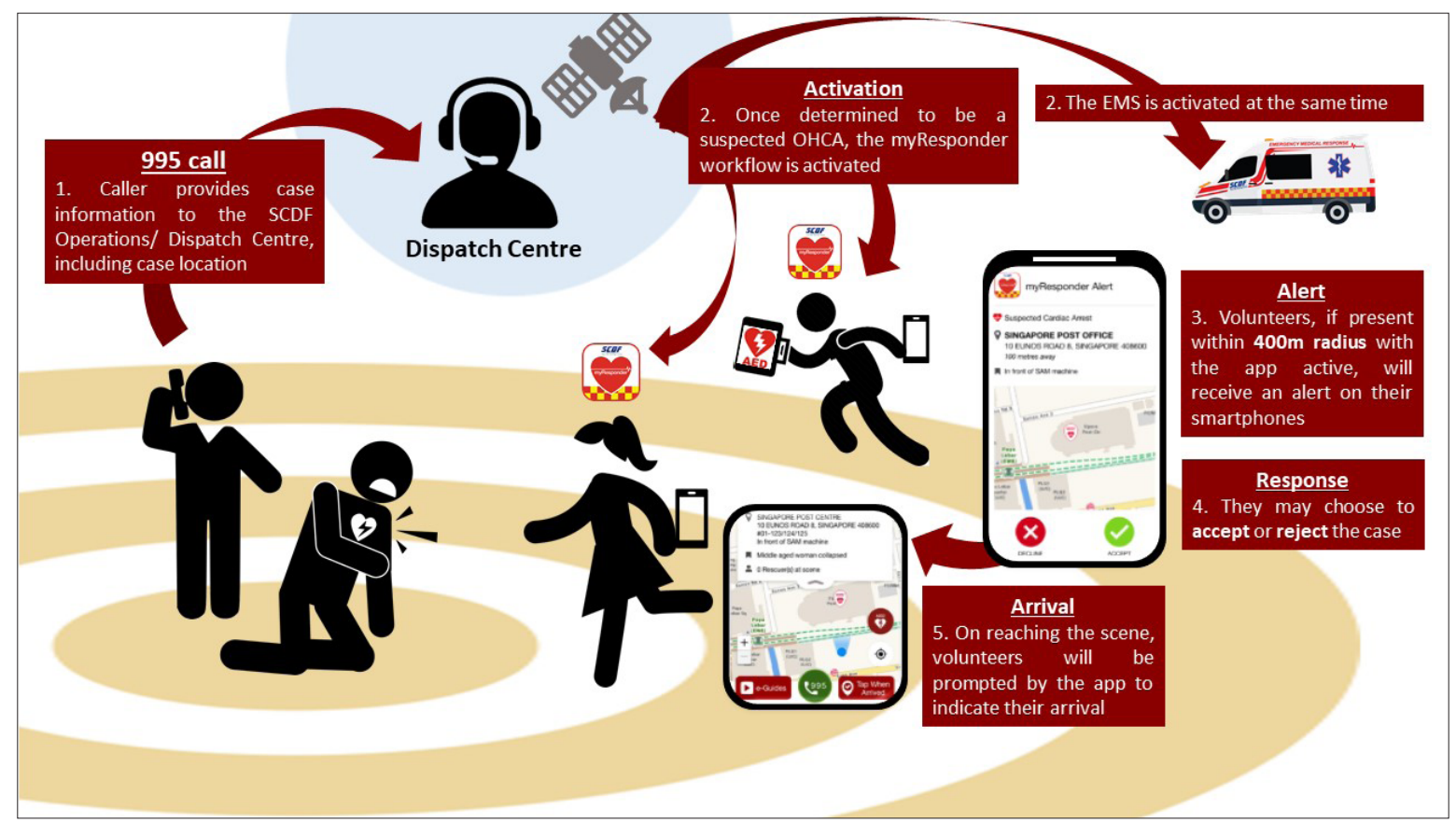

Fig 1. myResponder mobile application workflow.

\section{PAROS registry and myResponder database}

The Pan-Asian Resuscitation Outcome Study (PAROS) is an Asia-Pacific cardiac arrest registry set up in 2009 to understand OHCA and improve survival in Asia. ${ }^{12}$ It includes Singapore records of all OHCA attended by the SCDF EMS, including those sent to public hospitals, patient characteristics, incident location information, EMS dispatch information, as well as pre-hospital and emergency department resuscitation information. In this study, de-identified PAROS records from Singapore between April 2010 and December 2017 were used.

The myResponder database contains app-related records of all suspected OHCA in which the app was activated, including "activation", "alerts", "response", and "arrival" information. Although myResponder was implemented in April 2015, records of cases were only available from January 2016. For this study, de-identified records between January 2016 and December 2017 were used.

\section{Study design and outcomes}

This retrospective cohort study examined OHCA cases and outcomes in Singapore from 1 April 2010 to 31 December 2017. In this study, Period I corresponds to the baseline period from 1 April 2010 to 30 June 2012, where there were no significant interventions implemented to increase B-CPR. Period II corresponds to the period after implementation of DA-CPR (1 July 2012 to 31
March 2015), while Period III corresponds to the period with both DA-CPR and myResponder (1 April 2015 to 31 December 2017).

The primary exposure was the implementation of the respective interventions in each period. The primary outcome was B-CPR rate. B-CPR is defined as CPR initiated by any individual including passers-by, family members or off-duty healthcare professionals; it excludes on-duty EMS first responders, on-duty law enforcement officers and on-duty medical staff.

The secondary outcomes were survival-to-hospital discharge, and Utstein survival. Survival-to-hospital discharge in this study is defined as being discharged alive from hospital or remaining alive in hospital 30 days post-arrest. Utstein survival is a standardised format that facilitates reporting of OHCA resuscitation outcomes for comparison with other studies and represents cases where there are opportunities for intervention to improve survival outcomes. It is thus a consensus measurement of EMS system efficacy. To determine Utstein survival, only cases that are bystander-witnessed and presented with a shockable rhythm were included in the analysis ${ }^{13}$.

\section{Inclusion criteria}

Both adult and paediatric cases were included in this study. Cases with no prehospital resuscitation attempted, not attended to by the EMS, or that were EMS-witnessed, were excluded from the study. Cases with missing data for any study variable were also excluded. 


\section{Ethics approval}

SingHealth Centralised Institutional Review Board and National Healthcare Group Domain Specific Review Board granted approval with waiver of patient's informed consent for this study.

\section{Statistical methods}

Data analysis was carried out using software $\mathrm{R}$ version 3.6.1 for all statistical analysis. There were no missing data for any of the primary and secondary outcomes, as well as other relevant variables included in the analysis.

Univariate analyses were performed to compare the characteristics and survival outcomes of OHCA cases in the 3 periods. Chi-square test was used for categorical variables, and one-way ANOVA for continuous variables.

B-CPR rates from 1 April 2010 to 31 December 2017 were plotted as a time-series to visualise the differences in B-CPR rates over time. The primary and secondary hypotheses of increased B-CPR, survival-to-hospital discharge, and Utstein survival after the implementation of DA-CPR and myResponder, were tested using a segmented regression analysis of time series data. This analysis allows us to determine the impact each intervention has on B-CPR rates immediately and cumulatively over time, while accounting for baseline trends. ${ }^{14,15}$ It also allows us to circumvent the limitation of possible underreporting of B-CPR contributed by myResponder as the database relies on volunteers reporting their arrival, which may be inaccurate. By comparing all cases before and after myResponder intervention, we can determine myResponder's impact on our outcomes over time without requiring arrival information for each case. We recoded 5 variables on a month-on-month basis for 2 interventions (namely, DACPR and myResponder) to accommodate the segmented regression framework. The framework comprises baseline trend (a continuous variable representing time since the beginning of the observation period); immediate or "level" effect of each intervention (coded 0 before the intervention and 1 after the intervention); and rate of increase or "trend" effect of each intervention (coded 0 before the start of the intervention and coded as a continuous variable after the intervention). Multivariable logistic regression was performed with these variables included, adjusted for predictors that were significant at $P$ value of $<0.20$ based on a univariate analysis. Statistical significance was set at $P<0.05$.

\section{RESULTS}

A total of 15,355 OHCA cases occurred from April 2010 to December 2017, with 13,829 cases included in our analysis after excluding cases with no prehospital resuscitation, not EMS-attended, and EMS-witnessed. A small number $(n=6)$ with incomplete hospital outcomes were also excluded (Fig. 2). We compared the characteristics and outcomes of OHCA cases that occurred during the 3 periods (Table 1). Mean age of patients increased across the 3 time periods (63.6 versus 65.7 vs 67.1, $P<0.001)$. A greater proportion of cases were witnessed by bystanders $(53.7 \%$ vs $56.2 \%$ vs $59.9 \%, P<0.001)$ and occurred in residential locations over time $(72.9 \%$ vs $73.9 \%$ vs $75.6 \%, P<0.001)$. Proportion of cases with shockable rhythm $(19.2 \%$ vs $17.5 \%$ vs $16.1 \%, P<0.001)$ and a presumed cardiac aetiology (75.5\% vs $69.3 \%$ vs $66.6 \%, P<0.001)$ decreased over time. There was an increase in the proportion of cases with B-CPR $(24.8 \%$ vs $50.8 \%$ vs $64.4 \%, P<0.001)$, with bystander AED (1.8\% vs $3.3 \%$ vs $5.5 \%, P<0.001)$, survival-to-hospital discharge $(2.6 \%$ vs $3.7 \%$ vs $4.8 \%, P<0.001)$, and Utstein survival ( $12.4 \%$ vs $16.0 \%$ vs $21.6 \%, P<0.001)$ over time.

We plotted the trendline for yearly B-CPR rates from January 2010 to December 2017 (Fig. 3). B-CPR rates are shown in this diagram to account for the increasing number of OHCA cases over time; yearly B-CPR rates were calculated by dividing the number of cases recorded to have received $\mathrm{B}-\mathrm{CPR}$ over the total number of $\mathrm{OHCA}$ cases that occurred in that year. We noted an increase in B-CPR rates in 2012 after DA-CPR was introduced, followed by a gradual increase in B-CPR rates that appear to be plateauing over time. In addition, both the absolute number of OHCA cases and proportion of cases with B-CPR over the years have increased, in keeping with Singapore's ageing population. Similarly, cases associated with DA-CPR and myResponder have also increased over the years since their respective year of implementation.

Table 2 shows the segmented regression analysis for B-CPR, survival-to-hospital discharge, and Utstein survival after adjusting for underlying survival trends and other known predictors. Period II was significantly associated with an increase in odds of receiving B-CPR both immediately after the implementation of DA-CPR (level odds ratio [OR] 2.26, 95\% confidence interval [CI] 1.79-2.88, $P<0.001)$ and in the month-to-month trend (trend OR 1.03, 95\% CI: 1.01-1.04, $P=0.006)$. No level change was detected in Period III (OR 0.95, 95\% CI 0.82-1.11, $P=0.52$ ), while a slight reduction in rate of increase was noted, in line with our observed trend of positive but plateauing B-CPR rates (OR 0.99, 95\% CI 0.98-1.00, $P=0.04$ ). On the other hand, there were no changes in the adjusted 


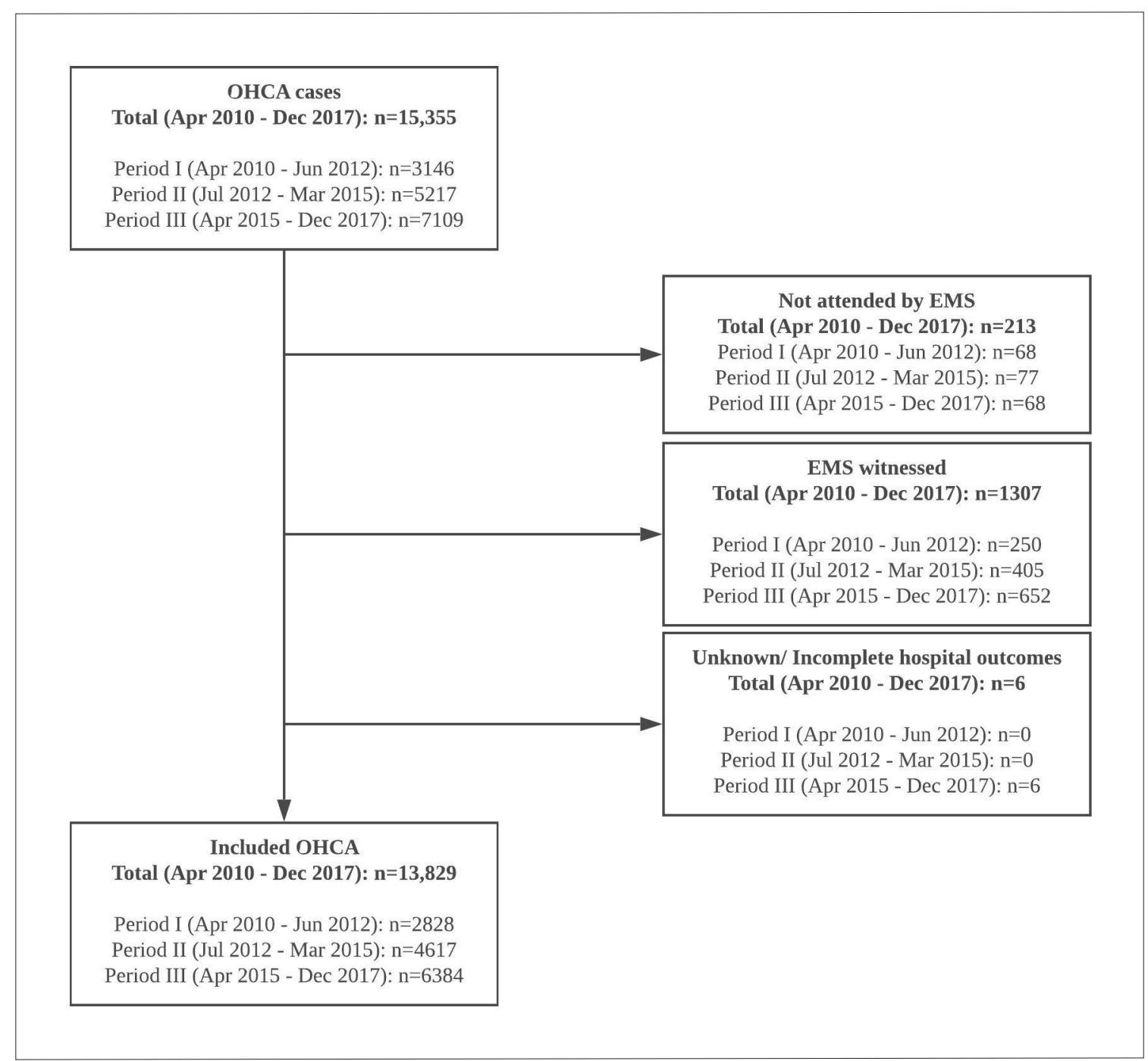

Fig 2. Inclusion criteria.

EMS: emergency medical service; OHCA: out-of-hospital cardiac arrest

odds of survival-to-hospital discharge in both Period II (level OR 0.85, 95\% CI 0.46-1.62, $P=0.61$; trend OR $0.98,95 \%$ CI $0.93-1.03, P=0.47)$ and Period III (level OR 1.21, 95\% CI 0.82-1.40, $P=0.34$; trend OR $0.99,95 \%$ CI $0.97-1.01, P=0.30$ ). Similarly, for Utstein survival, adjusted odds ratio showed no significant level and trend change in both Period II (level OR $1.03,95 \%$ CI $0.30-4.33, P=0.96$; trend OR $0.87,95 \%$ CI $0.74-1.01, P=0.08$ ) and III (level OR 1.76 , $95 \%$ CI $0.85-3.76, P=0.13$; trend OR $1.00,95 \%$ CI $0.96-1.04, P=0.94)$.

\section{DISCUSSION}

This retrospective cohort study was designed to examine the effect of 2 community-level interventions on B-CPR and survival over time.
Our study showed that B-CPR rates had improved over the 3 time periods, from $23.1 \%$ in 2010 to $67.3 \%$ in 2017. A visual inspection of yearly B-CPR rates showed an increasing but plateauing trend. Up-to-date literature trending B-CPR rates elsewhere is limited. In the US, a rising trend is similarly noted, reaching up to $43.6 \%$ in 2015. ${ }^{16}$ In Sweden, B-CPR rates increased from $46 \%$ to $73 \%$ from 1990 to $2009 .{ }^{17}$ In a previous study comparing OHCA outcomes from 7 countries under the PAROS network, B-CPR rate varied from $10.5 \%$ to $40.9 \%$ in the period between 2009 and 2012. ${ }^{18}$ These suggest that good progress has been made in improving B-CPR rates in Singapore over the years.

Specifically, we noted that the implementation of DA-CPR correlated with a positive level and trend change in the odds of receiving $\mathrm{B}-\mathrm{CPR}$, while no change 
Table 1. Comparison of clinical characteristics of OHCA cases

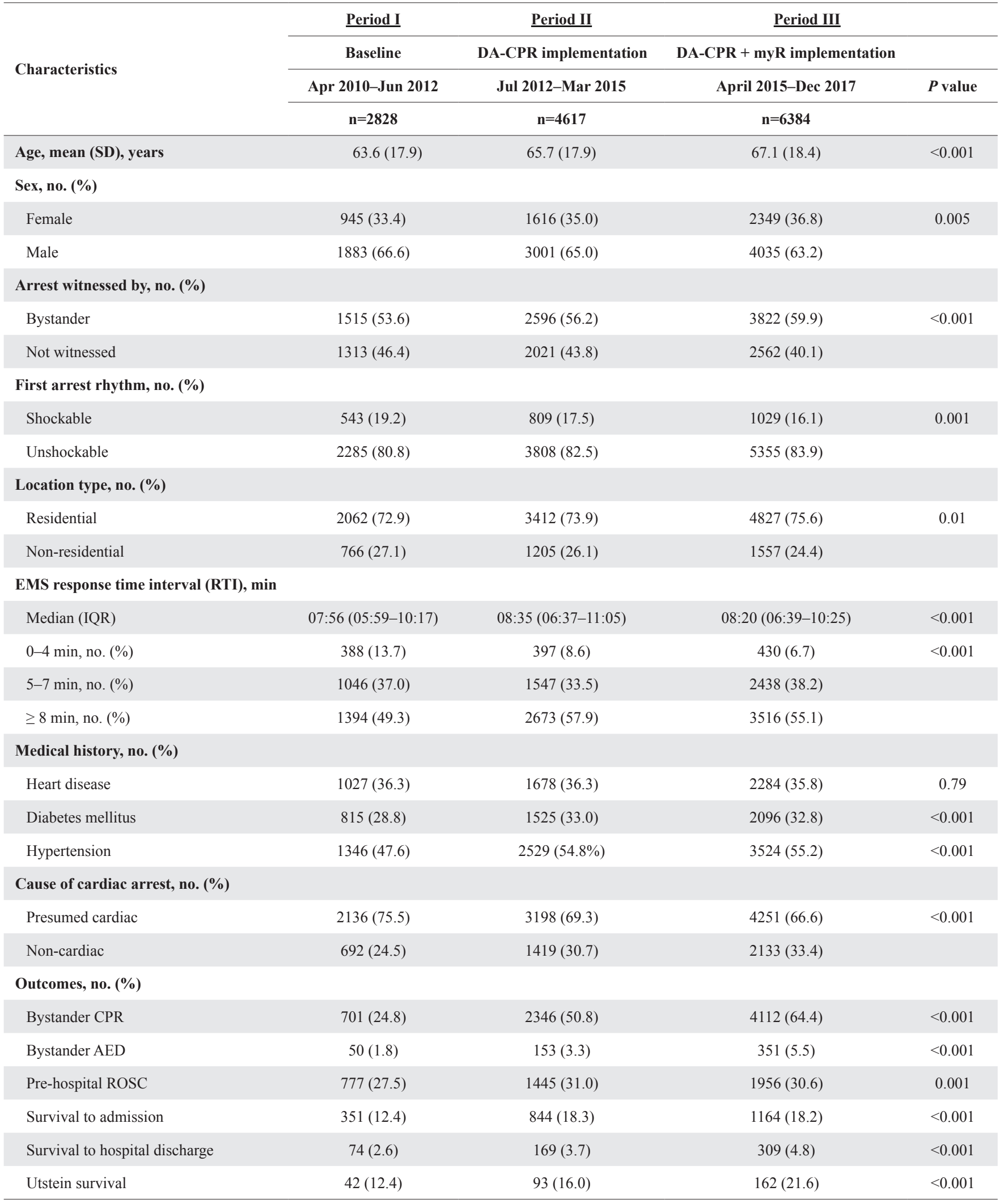

AED: automated external defibrillator; DA-CPR: dispatcher-assisted cardiopulmonary resuscitation; myR: myResponder; ROSC: return of spontaneous circulation 


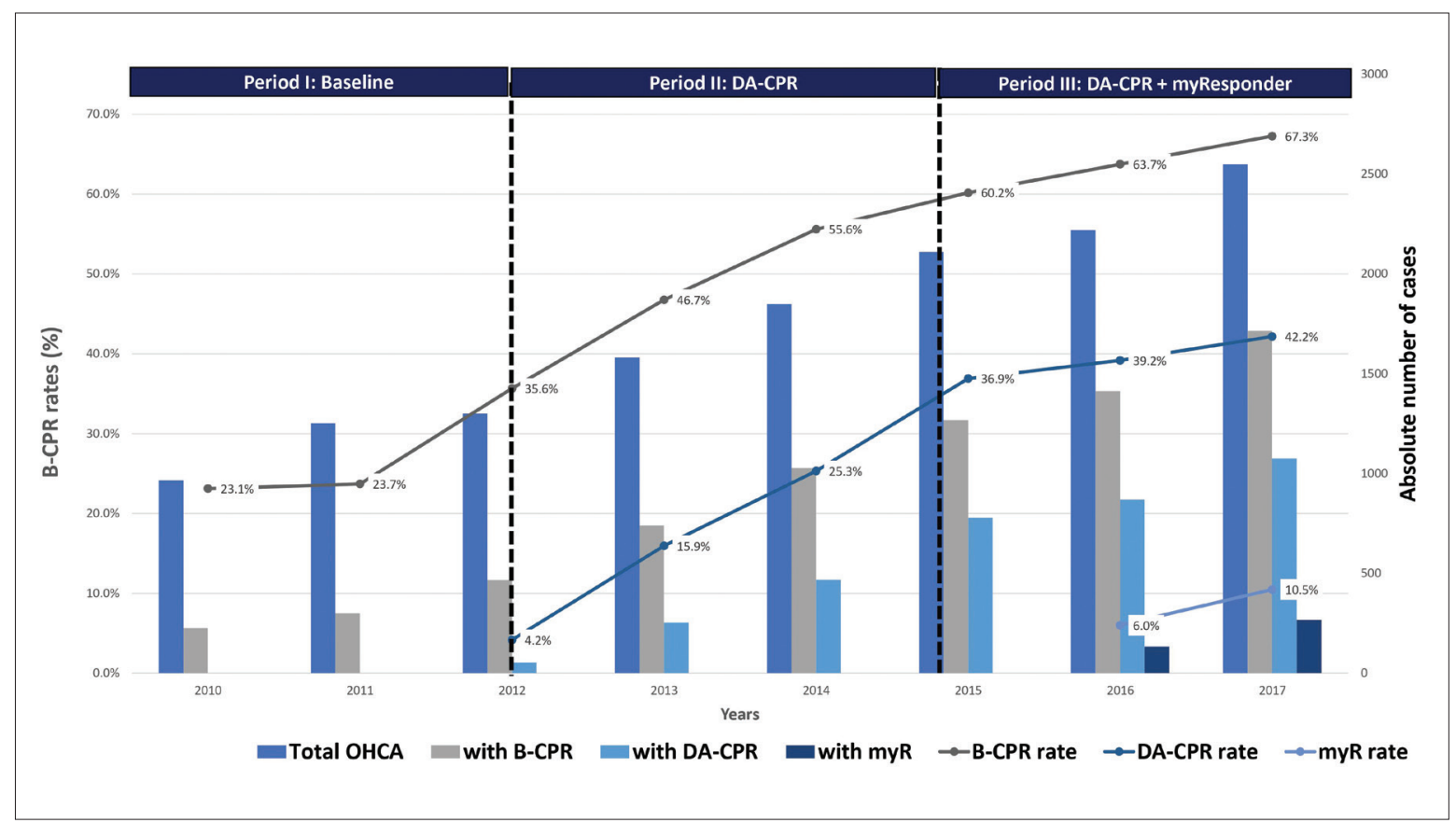

Fig 3. Time series of bystander CPR (B-CPR) rates from 2010 to 2017.

Time series of bystander CPR rates are indicated by solid black line. Also shown are the percentages of OHCA cases with interventions reported (DA-CPR rate and myR rate). In the clustered columns are the absolute number of cases associated with each intervention. For myResponder, data were only available starting from January 2016.

OHCA: out-of-hospital cardiac arrest; DA-CPR: dispatcher-assisted CPR; myR: myResponder mobile application

in level and a slight reduction in rate of increase was observed with myResponder implementation.

Several studies from other countries have shown that the implementation of community-level interventions were associated with improved bystander CPR rates, although the interventions evaluated in each of these studies differed. ${ }^{5,19,20}$ Other studies have evaluated the individual effect of DA-CPR and mobile phone dispatch services on $\mathrm{B}-\mathrm{CPR}$ rates; the positive association between DA-CPR and B-CPR rates is well-studied, ${ }^{21-23}$ while a randomised controlled trial in Stockholm showed that a mobile app similar to myResponder was significantly associated with increased rates of B-CPR. ${ }^{24}$

Our unpublished descriptive analyses revealed that a combination of factors-including a plateauing trend in DA-CPR; potential implementation lag time and lack of optimisation of myResponder that resulted in lower-thanexpected activation rates; and a substantial overlap in cases with both DA-CPR and myResponder-may explain the plateauing trend in B-CPR rates and why the implementation of myResponder was not associated with any increased odds of B-CPR.

Meanwhile, other studies have reported variable survival outcomes after the implementation DA$\mathrm{CPR},{ }^{25}$ with a systematic review reporting limited evidence in improvement of survival associated with this intervention. ${ }^{26}$ For example, higher survival rates were reported in King County (OR 1.45, 95\% CI $1.21-1.73)^{22}$ and Arizona (OR 1.5, 95\% CI 1.1-2.1) in the US, ${ }^{25}$ while a reduction was seen in a study in Ottawa in Canada $(4.8 \%$ in control period to $3.0 \%$ after intervention). ${ }^{23}$ On the other hand, a text message alert system to activate trained volunteer to OHCA cases in the Netherlands was associated with increased survival-to-hospital discharge (OR 2.82, $95 \%$ CI $1.52-5.24) .{ }^{27}$

Several reasons may explain why we did not observe statistical significance for survival with both interventions. Firstly, the presence of B-CPR alone may not translate to improved survival if high-quality CPR is not administered. Both DA-CPR and myResponder do not track the quality of B-CPR done on patients, nor do they require laypersons to have valid certifications in CPR training before they are instructed to do DA-CPR or are dispatched to an OHCA scene as a myResponder volunteer. It is likely that patients had received B-CPR of variable quality with our interventions. For myResponder, it is conceivable that in some cases, volunteers were unable to arrive substantially early enough to improve the patient's chance of survival. 
Table 2. Adjusted odds ratio comparing B-CPR, survival-to-hospital discharge, and Utstein survival

\begin{tabular}{|c|c|c|c|c|c|c|}
\hline & \multicolumn{2}{|c|}{$\mathbf{B}-\mathbf{C P R}^{\mathrm{a}}$} & \multicolumn{2}{|c|}{ Survival to hospital discharge ${ }^{\mathrm{b}}$} & \multicolumn{2}{|c|}{ Utstein survival $^{\mathrm{c}}$} \\
\hline & $\begin{array}{c}\text { Adjusted OR } \\
\text { (95\% CI) }\end{array}$ & $P$ value & $\begin{array}{c}\text { Adjusted OR } \\
\text { (95\% CI) }\end{array}$ & $P$ value & $\begin{array}{c}\text { Adjusted OR } \\
\text { (95\% CI) }\end{array}$ & $P$ value \\
\hline \multicolumn{7}{|l|}{ Period I (10 Apr-12 Jun) } \\
\hline $1^{\text {st }}$ month of period (10 Apr) & 1.00 & & 1.00 & & 1.00 & \\
\hline Trend change & $1.00(0.98-1.02)$ & 0.86 & $1.03(0.98-1.08)$ & 0.21 & $1.14(0.99-1.35)$ & 0.09 \\
\hline \multicolumn{7}{|l|}{ Period II (12 Jul-15 Mar) } \\
\hline Period I (10 Apr- 12 Jun) & 1.00 & & 1.00 & & 1.00 & \\
\hline Level change & $2.26(1.79-2.88)$ & $<0.001^{\mathrm{d}}$ & $0.85(0.46-1.62)$ & 0.61 & $1.03(0.30-4.33)$ & 0.96 \\
\hline Trend change & $1.03(1.01-1.04)$ & $0.006^{\mathrm{d}}$ & $0.98(0.93-1.03)$ & 0.47 & $0.87(0.74-1.01)$ & 0.08 \\
\hline \multicolumn{7}{|l|}{ Period III (15 Apr-17 Dec) } \\
\hline Period I to II (10 Apr- 15 Mar) & 1.00 & & 1.00 & & 1.00 & \\
\hline Level change & $0.95(0.82-1.11)$ & 0.52 & $1.21(0.82-1.80)$ & 0.34 & $1.76(0.85-3.76)$ & 0.13 \\
\hline Trend change & $0.99(0.98-1.00)$ & $0.04^{\mathrm{d}}$ & $0.99(0.97-1.01)$ & 0.30 & $1.00(0.96-1.04)$ & 0.94 \\
\hline \multicolumn{7}{|l|}{ Other Predictors } \\
\hline Sex & - & - & $0.98(0.78-1.24)$ & 0.89 & - & - \\
\hline Age & - & - & $1.69(1.38-2.08)$ & $<0.00^{\mathrm{d}}$ & $1.66(1.16-2.39)$ & $0.006^{\mathrm{d}}$ \\
\hline Location & $1.79(1.64-1.02)$ & $<0.001^{\mathrm{d}}$ & $2.09(1.72-2.54)$ & $<0.001^{\mathrm{d}}$ & $2.17(1.51-3.11)$ & $<0.001^{\mathrm{d}}$ \\
\hline Witnessed & $1.26(1.17-1.02)$ & $<0.001^{\mathrm{d}}$ & $2.03(1.62-2.57)$ & $<0.001^{\mathrm{d}}$ & - & - \\
\hline Rhythm & - & - & $8.74(7.03-10.92)$ & $<0.001^{\mathrm{d}}$ & - & - \\
\hline Response time interval & - & - & $1.49(1.24-1.79)$ & $<0.001^{\mathrm{d}}$ & $1.32(0.93-1.87)$ & 0.11 \\
\hline Aetiology & - & - & $0.64(0.51-0.82)$ & $<0.001^{\mathrm{d}}$ & $2.05(1.45-2.90)$ & $<0.001^{\mathrm{d}}$ \\
\hline
\end{tabular}

${ }^{\mathrm{a}}$ Adjusted for location and witness status. Sex, age and aetiology were excluded from the multivariable logistic regression.

${ }^{\mathrm{b}}$ Adjusted for sex, age, location, witness status, rhythm, response time interval and aetiology.

${ }^{\mathrm{c}}$ Adjusted for age, location, response time interval and aetiology. Sex was excluded from the multivariable logistic regression.

d $P$ value $<0.05$

Furthermore, only early CPR was assessed in this study; the effects of other links in the chain of survival such as public AED utilisation rates (rapid defibrillation), management by the EMS (basic and advanced EMS), and management at the emergency department and hospital (advanced life support and post-cardiac arrest care) were not evaluated.

\section{Limitations and future research}

As an observational study, we are unable to establish the causative relationship between our interventions and the increase in B-CPR rates over time. The Save-A-Life (SAL) initiative, an intervention piloted shortly after myResponder in July $2015,{ }^{28}$ was not explicitly accounted for in our segmented regression analysis. This intervention involved free, standardised CPR and AED training to members of the public, as well as installation of publicly accessible AEDs at the ground floor of government housing estates. Also, the DispatcherAssisted first REsponder (DARE) programme in Singapore was introduced in April 2014 as a simplified CPR/AED course for members of public to gain skills and confidence in performing compression-only CPR and using the AED under the instructions of a dispatcher. Both interventions were not identified as separate segments in our analysis as public education on CPR/AED, in different formats, has been ongoing throughout the observation period. Hence, the impact of public education as a whole could likely have contributed to the increasing B-CPR trend, although we are unable to quantify this due the limitation of our study's methodology. Nonetheless, we note that there was no 
clear change in level or gradient at the time of implementation of both SAL and DARE on visual inspection of the month-on-month trendline for B-CPR rates. We were also unable to evaluate our interventions' impact on the long-term quality of life and functional outcome of the OHCA patients, due to missing data at the point of writing.

Records of time of arrival of myResponder bystanders were available but could be inaccurate as it requires the responder to indicate their own arrival, which they may not have done while being engaged at the scene. For $\mathrm{B}-\mathrm{CPR}$ related to myResponder, additional in-app functions, such as geo-fencing, may help to better track responders' timeliness.

Future studies could be carried out to address some of the trends observed in this paper. To assess the plateauing $\mathrm{B}-\mathrm{CPR}$ trend, a focused analysis on cases with no $\mathrm{B}-\mathrm{CPR}$ can be conducted to examine the common factors that might explain the absence of B-CPR in these cases and to understand why our interventions had limited impact on these cases. We may also perform analyses to evaluate how myResponder can be further optimised to improve both B-CPR rates and survival. The possibility of myResponder augmenting B-CPR in nursing homes could be studied; a recent study suggests that a proportion of nursing home residents may have received inadequate resuscitation despite trained $\mathrm{NH}$ staff. ${ }^{29}$ In addition, audiovisual CPR feedback appears to improve the quality of CPR during training ${ }^{30}$ and the incorporation of a similar feedback function into myResponder could be considered. Lastly, as prehospital defibrillation significantly reduces mortality, ${ }^{31}$ we can also explore how myResponder may be optimised to enable community responders to retrieve nearby AEDs more efficiently. Findings from these additional studies may help us to fine-tune these ongoing interventions to improve B-CPR rates and survival outcomes.

\section{CONCLUSION}

B-CPR rates in Singapore have shown an increasing trend alongside the implementation of communitylevel interventions such as DA-CPR and myResponder. The implementation of DA-CPR was associated with improved odds of receiving B-CPR over time while the impact of myResponder was less clear. A focus on high-quality CPR by laypersons and retrieval of AED via myResponder are potential future strategies to improve survival outcomes. Future studies are needed to better understand the plateauing B-CPR trend and to identify ways to optimise survival.

\section{Acknowledgements}

The authors would like to thank the late Ms Susan Yap and Ms Maeve Pek from Department of Emergency Medicine, Singapore General Hospital; Ms Noor Azuin, Ms Nurul Asyikin and all Medical Dispatcher Specialists from Unit for Prehospital Emergency Care, Singapore General Hospital for their contributions and support to the Singapore OHCA registry, and all other PAROS investigators that contributed to the study. The authors also would like to thank all EMS Medical Dispatchers from Singapore Civil Defence Force for their efforts in implementing dispatcher-assisted CPR.

\section{REFERENCES}

1. Berdowski J, Berg RA, Tijssen JG, et al. Global incidences of out-of-hospital cardiac arrest and survival rates: Systematic review of 67 prospective studies. Resuscitation 2010;81:1479-87.

2. White AE WW, Ong MEH. 2011-2015 Singapore out-of-hospital cardiac arrest registry report. 2017.

3. Nolan J, Soar J, Eikeland H. The chain of survival. Resuscitation 2006;71:270-1.

4. Larsen MP, Eisenberg MS, Cummins RO, et al. Predicting survival from out-of-hospital cardiac arrest: A graphic model. Ann Emerg Med 1993;22:1652-8.

5. Hasselqvist-Ax I, Riva G, Herlitz J, et al. Early cardiopulmonary resuscitation in out-of-hospital cardiac arrest. N Engl J Med 2015;372:2307-15.

6. Song J, Guo W, Lu X, et al. The effect of bystander cardiopulmonary resuscitation on the survival of out-of-hospital cardiac arrests: A systematic review and meta-analysis. Scand J Trauma Resusc Emerg Med 2018;26:86.

7. Population and population structure, 27 Sep 2018. Available at: https://www.singstat.gov.sg/find-data/search-by-theme/population/ population-and-population-structure/latest-data. Accessed on 10 August 2019.

8. Fire, ambulance and enforcement statistics 2017. Available at: https://www.scdf.gov.sg/docs/default-source/scdf-library/publications/ amb-fire-inspection-statistics/fire-ambulance-enforcementstatistics-2017.pdf. Accessed on 10 August 2019.

9. Kurz MC, Bobrow BJ, Buckingham J, et al. Telecommunicator cardiopulmonary resuscitation: A policy statement from the American Heart Association. Circulation 2020;141:e686-e700.

10. Florence D, Thomas DR, Carol F, et al. Chest compression alone cardiopulmonary resuscitation is associated with better long-term survival compared with standard cardiopulmonary resuscitation. Circulation 2013;127:435-41.

11. Harjanto S, Na MX, Hao Y, et al. A before-after interventional trial of dispatcher-assisted cardio-pulmonary resuscitation for out-of-hospital cardiac arrests in singapore. Resuscitation 2016;102:85-93.

12. Ong ME, Shin SD, Tanaka H, et al. Rationale, methodology, and implementation of a dispatcher-assisted cardiopulmonary resuscitation trial in the Asia-Pacific (Pan-Asian Resuscitation Outcomes Study phase 2). Prehosp Emerg Care 2015;19:87-95.

13. Perkins GD, Jacobs IG, Nadkarni VM, et al. Cardiac arrest and cardiopulmonary resuscitation outcome reports: Update of the Utstein resuscitation registry templates for out-of-hospital cardiac arrest: A statement for healthcare professionals from a task force of the International Liaison Committee on Resuscitation (American Heart 
Association, European Resuscitation Council, Australian and New Zealand Council on Resuscitation, Heart and Stroke Foundation of Canada, Interamerican Heart Foundation, Resuscitation Council of Southern Africa, Resuscitation Council of Asia); and the American Heart Association Emergency Cardiovascular Care Committee and the Council on Cardiopulmonary, Critical Care, Perioperative and Resuscitation. Resuscitation 2015;96:328-40.

14. Wagner AK, Soumerai SB, Zhang F, et al. Segmented regression analysis of interrupted time series studies in medication use research. J Clin Pharm Ther 2002;27:299-309.

15. Deasy C, Bray JE, Smith K, et al. Cardiac arrest outcomes before and after the 2005 resuscitation guidelines implementation: Evidence of improvement? Resuscitation 2011;82:984-8.

16. Virani SS, Alonso A, Benjamin EJ, et al. Heart disease and stroke statistics-2020 update: A report from the American Heart Association. Circulation 2020;141:e139-e596.

17. Adielsson A, Hollenberg J, Karlsson T, et al. Increase in survival and bystander CPR in out-of-hospital shockable arrhythmia: Bystander CPR and female gender are predictors of improved outcome. Experiences from sweden in an 18-year perspective. Heart 2011; 97:1391-6.

18. Ong ME, Shin SD, De Souza NN, et al. Outcomes for out-of-hospital cardiac arrests across 7 countries in asia: The Pan Asian Resuscitation Outcomes Study (PAROS). Resuscitation. 2015;96:100-8

19. Wissenberg M, Lippert FK, Folke F, et al. Association of national initiatives to improve cardiac arrest management with rates of bystander intervention and patient survival after out-of-hospital cardiac arrest. JAMA 2013;310:1377-84.

20. Malta Hansen C, Kragholm K, Pearson DA, et al. Association of bystander and first-responder intervention with survival after out-of-hospital cardiac arrest in North Carolina, 2010-2013. JAMA 2015;314:255-64

21. Culley LL, Clark JJ, Eisenberg MS, et al. Dispatcher-assisted telephone CPR: Common delays and time standards for delivery. Ann Emerg Med 1991;20:362-6.
22. Rea TD, Eisenberg MS, Culley LL, et al. Dispatcher-assisted cardiopulmonary resuscitation and survival in cardiac arrest. Circulation. 2001;104:2513-6.

23. Vaillancourt C, Verma A, Trickett J, et al. Evaluating the effectiveness of dispatch-assisted cardiopulmonary resuscitation instructions. Acad Emerg Med 2007;14:877-83.

24. Ringh M, Rosenqvist M, Hollenberg J, et al. Mobile-phone dispatch of laypersons for CPR in out-of-hospital cardiac arrest. N Engl J Med 2015;372:2316-25.

25. Bobrow B, Panczyk M, Stolz U, et al. Abstract 1: Statewide implementation of a standardized prearrival telephone CPR program is associated with increased bystander CPR and survival from out-ofhospital cardiac arrest. Circulation 2014;130:A1-A.

26. Bohm K, Vaillancourt $\mathrm{C}$, Charette $\mathrm{ML}$, et al. In patients with out-of-hospital cardiac arrest, does the provision of dispatch cardiopulmonary resuscitation instructions as opposed to no instructions improve outcome: A systematic review of the literature. Resuscitation 2011;82:1490-5.

27. Pijls RWM, Nelemans PJ, Rahel BM, et al. A text message alert system for trained volunteers improves out-of-hospital cardiac arrest survival. Resuscitation 2016;105:182-7.

28. Tay PJM, Pek PP, Fan Q, et al. Effectiveness of a community based out-of-hospital cardiac arrest (OHCA) interventional bundle: Results of a pilot study. Resuscitation 2020;146:220-8.

29. Ho AF, Lee KY, Lin X, et al. Nation-wide observational study of cardiac arrests occurring in nursing homes and nursing facilities in Singapore. Ann Acad Med Singap 2020;49:285-93.

30. Wee JC, Nandakumar M, Chan YH, et al. Effect of using an audiovisual CPR feedback device on chest compression rate and depth. Ann Acad Med Singap 2014;43:33-8.

31. Tay WJ, Li H, Ho AF, et al. Mortality and neurological outcomes in out-of-hospital cardiac arrest patients with and without targeted temperature management in a multiethnic asian population. Ann Acad Med Singap 2020;49:127-36 